Article

\title{
An Experimental Study on the Influence of Soundscapes on People's Behaviour in an Open Public Space
}

\author{
Francesco Aletta ${ }^{1}$, Federica Lepore ${ }^{2}$, Eirini Kostara-Konstantinou ${ }^{2}$, Jian Kang ${ }^{1, *}$ \\ and Arianna Astolfi ${ }^{2}$ \\ 1 School of Architecture, University of Sheffield, Sheffield S102TN, UK; f.aletta@sheffield.ac.uk \\ 2 Department of Energy, Politecnico di Torino, Torino 10129, Italy; federica.lepore@studenti.polito.it (F.L.); \\ eirini.kostarakonstantinou@studenti.polito.it (E.K.-K.); arianna.astolfi@polito.it (A.A.) \\ * Correspondence: j.kang@sheffield.ac.uk; Tel.: +44-114-222-0325
}

Academic Editor: Gino Iannace

Received: 25 July 2016; Accepted: 20 September 2016; Published: 27 September 2016

\begin{abstract}
Several studies have investigated how environmental sounds and music can modulate people's behaviours, particularly in marketing research. However, there are relatively few examples of research about such relationships with a focus on the management of urban public spaces. The current study investigated an open public space used mainly as a pedestrian crossing to analyse the relationship between the audio stimuli and peoples' behaviours. An experiment relying on covert behavioural observation was performed. During the experiment, three different music stimuli and a control condition (i.e., no music) were reproduced in order to find out firstly whether music compared to no music could elicit an increase in the number of people stopping in the investigated area, and secondly whether music is associated with a longer duration of stay for those who stop. Results showed that the presence of music had no effect on the number of people stopping in the area, but it had a statistically significant effect on the duration of stay for those who stopped. The above findings support the idea that people felt more invited to stay in the area with music rather than with no music, and suggest that the acoustical manipulation of the existing sound environment could provide soundscape strategies capable of promoting social cohesion in public spaces.
\end{abstract}

Keywords: soundscape; music stimuli; environmental noise; people's behaviour

\section{Introduction}

Since the 1970s, soundscape started to emerge as a new science and research field. Schafer and his colleagues defined this concept as "an environment of sound (or sonic environment) with emphasis on the way it is perceived and understood by the individual or a society" [1]. The International Organization for Standardization [2] released a standard in 2014 where soundscape is defined as the "acoustic environment as perceived or experienced and/or understood by a person or people, in context". The number of studies in the field is increasing over time, and there is more and more research interest on the relationships between sonic environments, people, and contexts.

Over the last decades, several studies have investigated the influence of environmental sounds and music on peoples' behaviour, especially in terms of the pace of some activities or performance, mainly with a commercial or business perspective. These studies considered the effects of sound on individuals' mood, music perception, and behaviours through a wide range of parameters, such as productivity in the workplace, pace of shopping, and time and money spent in aggregation places (e.g., restaurants and shopping malls). Regarding product choice, Areni and Kim [3] showed that classical music played in a wine shop resulted in consumers choosing more expensive wines. Generally, 
the results supported the idea that a good perceived match between music and context improves persuasion. Subsequently, Yalch and Spangenberg [4] focused their research on time spent in a retail context, and suggested that more familiar background music (vs. less familiar) decreased consumers' actual shopping time, and, conversely, unfamiliar music triggered consumers' attention.

Previously, Milliman [5] investigated the relationship between pace of shopping, sales, and tempo. His research suggested that slower music is related to a slower pace of peoples' movement in a retail environment, and, as a result, more time and money spent there; while fast musical tempo reproduced in a restaurant caused a shorter duration of eating time [6]. Focusing more on the effect of music tempo, Kampfe, Sedlmeier, and Renkewitz [7] asserted that hearing background music characterized by a fast tempo leads to an increase in the pace of certain behaviours, such as drawing, reading, walking and running, and eating and drinking. In general, there are two fundamental hypotheses for these effects; the first is the synchronization of the movements to the musical beat, while the second is the increase in the arousal found with fast-tempo music [8].

Even if several studies explored the effect of sound environment on consumers' behaviour (mainly observed in indoor contexts), there have been limited studies investigating its effects regarding the management of public urban spaces. There is an increasing interest about the effects of background sounds on listeners' behaviour, focusing on the effectiveness of soundscape strategies on the sustainable development of cities and the improvement of the health and the quality of life [9]. Witchel et al. [10] managed to improve crowd behaviour and to decrease anti-social behaviours through the manipulation of the outdoor acoustic environment with sounds and music played onto the main street of the city's busiest clubbing and entertainment district in Brighton and Hove (UK). Sayin et al. [6] reported a significant effect of human sounds and music on the perceived safety of individuals in public places that are characterized by people's reluctance and avoidance, such as car parks, metro stations, and many green areas. Finally, Thomas Schafer et al. [11] assessed the relationship between the processing of music and the experience of safety and danger and found that music scenarios are experienced as less stressful and dangerous than silent scenarios. In particular, silence or very repetitive and monotonous auditory stimuli representing absence of information about the surroundings might be expected to evoke feelings of stress or danger. While the study by Schafer et al. relied on a laboratory experiment, raising some issues about its ecological validity, it does show that music can modulate human perception and behaviours.

All of the above-mentioned research focusing on public spaces explored the potential of sound to decrease anti-social behaviour and negative feelings such as anxiety and lack of safety in public areas. According to Brown et al. [12], safety and sense of control are some of the outcomes included in the philosophy of soundscape. Communication, enjoyment, and comfort are other aspects underpinning the final soundscape construct. However, there is a current lack of research focusing on these latter aspects, and overall on the potential of the acoustic environment to support social cohesion in public spaces. The "safer, cleaner, greener" UK policy on "live-ability" [13] for well-designed and well-planned urban places was discussed by Dempsey [14], who investigated the relationship between a good-quality built environment and social cohesion through the identification of features capable of providing quality in the built environment. Accessibility, inclusiveness, connectedness, and permeability were some of the analysed features, contextualized within a neighbourhood.

The current research focused on the area under the Concourse Bridge, the improvement of which is one of the key projects of the long-term Masterplan proposed by the University of Sheffield and built upon the University Estates Strategy 2010-2015. The intention is to reinforce the link between the connected spaces and upgrade the quality of the area through a combination of traditional urban renewal practises based on visual aspects (e.g., light, greenery) [15]. In addition to this, the rationale for the current study was to test whether the sound—as a feature of the physical environment-would influence peoples' perception of a public place with no particular aesthetic and social dimension, through the introduction of music in order to create a more "desirable" public space and lead the end users to a satisfying experience without any kind of intervention on the existing buildings. 
In particular, the main aims of the study were: (1) to test whether there is an effect of added music sounds on the number of persons stopping in the selected public urban space; (2) to test whether there is an effect of added music sounds on people's duration of stay in the selected public urban space.

For this purpose, covert behavioural observations were performed under the Concourse Bridge, an open public space at the University of Sheffield (UK), mainly used for connection between different buildings within the University campus. Three different music excerpts and a control condition (no music) were cyclically reproduced over a term week at different times, and video-recording data were gathered and processed for behavioural observations [10]. To the knowledge of the authors, this is one of the first attempts in soundscape studies to manipulate an actual acoustic environment in an open public space, collecting data in a systematic way through a covert observational protocol.

\section{Materials and Methods}

This study relies on non-participant covert observations, during which the users-not aware of the experiment-were video-recorded, and the final subconscious and spontaneous responses constituted the database used for later behavioural analysis. Indeed, there is still no clear agreement on what is the optimal method for data collection in soundscape studies. In most cases, data collection is participative and participants are required to have an attentive listening style. According to Aletta et al. [16], this raises a question about whether being "aware" of taking part in a soundscape study might actually affect the responses people give. The study received ethic approval through the Ethics Committee procedure of the School of Architecture, University of Sheffield (ref: 002442, January 2015). Cameras were set to a low resolution in order to avoid the identification of peoples' faces.

\subsection{Study Area}

The analysed public space is inside the University campus, and serves as the connection between different Departments' facilities and the central building where services and administration offices, as well as bars, restaurants, and shops are located. It is a pedestrian area below a raised vehicular bridge, an important crossroad for pedestrians coming from Weston park area, the Alfred Denny Building, the Arts Tower, and the Western Bank Library toward the Hicks building, Students' Union, and the Octagon centre, and it has not undergone any particular design interventions over the past 15 years. Since then, different attempts have been made to substantially improve the public space and enhance the public dimension underneath the Concourse Bridge, which is made of raw concrete, mainly in dim light during the day and poorly lit at night, and hosts the highest pedestrian affluence across campus. The Concourse Bridge is a key student space providing an important link beneath Western Bank, offering space for shelter and gatherings [15]. It constitutes a node where people usually stop for a break between various activities within the campus. Given the centrality of the area, the space-divided by pylons into two branches—presents low solid parapets along the edges used like benches in order to facilitate people's stay. The main sound sources are human voices and the traffic coming from the upper arterial road of Western Bank. Figure 1 shows the location of the site, as well as the observation area and the location of the cameras, which will be better described in the following section.
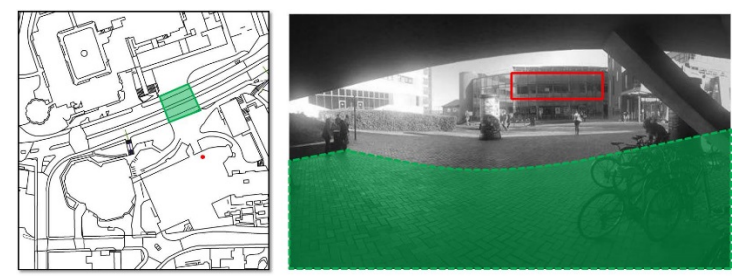

Figure 1. Plan of the site (left); the green area shows the observation area (underneath the Concourse Bridge); the red dot shows the location of the cameras used for video recordings in the Students' Union building (right)—See also details in Section 2.2. 


\subsection{Equipment and Procedure}

The experiment was performed during five consecutive working days in February 2015. The equipment used for the experiment was: a laptop with the driver software of the audio card for outputs regulation; a professional audio card (RME Babyface); two loudspeakers (Genelec 8030B); and two cameras (Sony handycam DCR-DVD115) for video recording.

The nature of a non-participant observational study implies that no interaction between the research team and the participants would happen. In fact, the two cameras were placed at the first floor of the Students' Union building in order to cover the investigated area, and were hidden from people's sight (as shown in Figure 1).

In order to characterise the acoustic environment of the observation area, a set of ten simultaneous ten-minute background noise level measurements were carried out, using ten $1 / 8^{\prime \prime}$ microphones (B\&K) connected to a portable recorder (Edirol), placed along the direction of walking at two-meter distance intervals. At the same time, a computation of the sound propagation in the area was made using two reference omnidirectional sound sources with a sound power of $100 \mathrm{~dB}$ (white noise spectrum) to simulate the loudspeakers to be installed for the experiment. The simulation of the sound propagation reported in Figure 2 shows a relatively homogeneous sound pressure levels (SPL) distribution in the observation area. If this information is compared with the background noise level profile, it can be reasonably assumed that people in the observation area would be approximately exposed to the same sound field, regardless of their location.

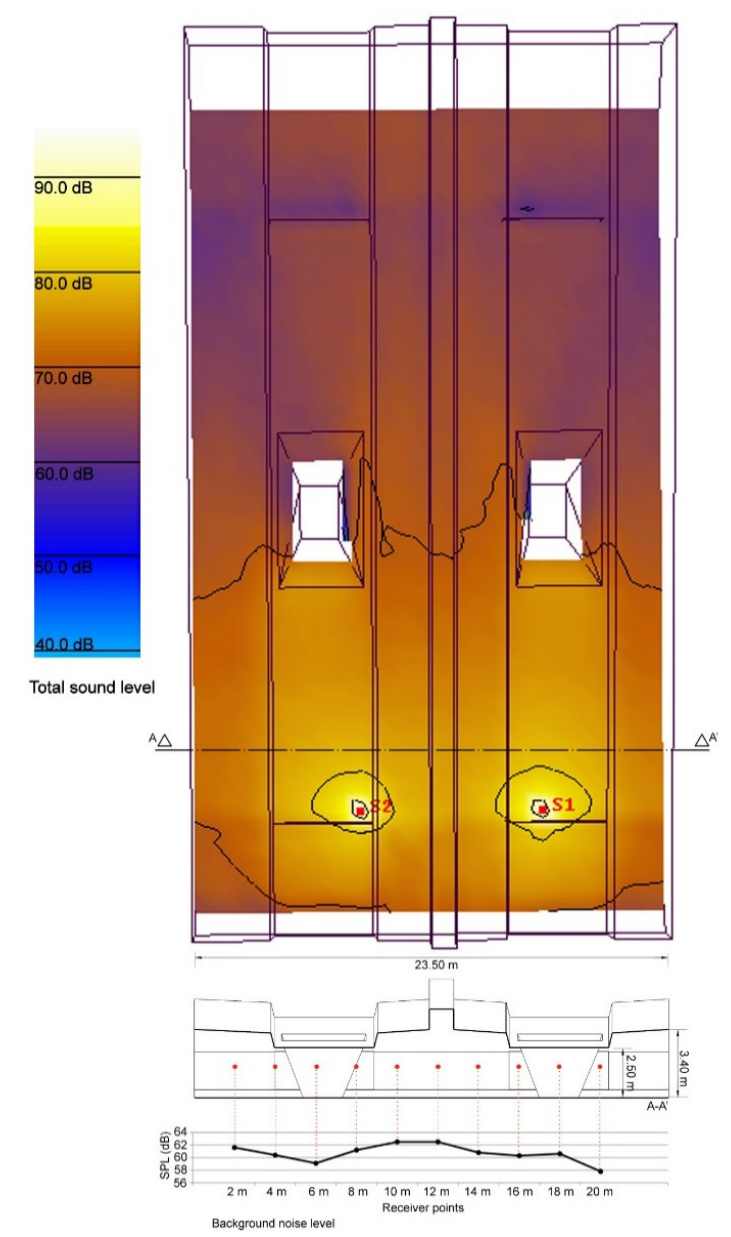

Figure 2. Sound pressure level (SPL) distribution simulation with location of the hypothetical sound sources (loudspeakers) and section with location of the microphones and corresponding background noise levels. 


\subsection{Signal Analysis}

During the experiment, a playlist of three music excerpts and a control condition (no music) was cyclically reproduced. Following the protocol of a previous experiment carried out by Witchel and colleagues [10], three instrumental pieces of music from contrasting genres (namely classical, jazz, and ambient electronic music) were used to ensure a high level of inclusiveness, involving different social groups in terms of age and gender [17]. It has been affirmed that conventional acoustic parameters for noise measurement-e.g., weighted sound pressure levels (SPLs) - are not sufficient for the adequate measurement of soundscape [18-22].

Since soundscape is a perceptual construct and music is known to trigger emotions and affect perception, soundscape and music are closely related; thus, the psychoacoustic parameters that have previously been applied mainly in music perception may also be applicable in soundscape research [23]. However, for the purpose of this study, it was decided to rely on pitch-related parameters for the selection of the stimuli to be used in the experiment. The pitch features are particularly significant in the field of environmental sounds and their perception [18]. Pitch maybe defined as "that attribute of auditory sensation in terms of which sounds maybe ordered on a musical scale from high to low", and is generally assigned by the frequency of a pure tone having the same subjective pitch as the considered sound [24,25]. Another additional parameter defining other pitch sensations is the pitch strength (PA, a-dimensional), corresponding to the assessment of the sound along a scale from faint to distinct. It has been asserted that high pitch could be associated with feelings like strong activation, excitement, surprise, happiness. Low pitch instead could be associated with low activation, calmness, and quietness [26-28]. According to Yang [21], the values of the most prominent pitches among environmental sounds are $4000 \mathrm{~Hz}$ for bird songs, $1000 \mathrm{~Hz}$ for fountain sounds, and no pitch perceived for river, sea waves, traffic, and wind. Western music generally refers to the $440 \mathrm{~Hz}$ pitch [29]. Therefore, the rationale for selecting the stimuli was having music excerpts with a relatively broad range of pitch features. These were selected amid those used in Witchel's experiment in Brighton [10]: Classical (Waltz of the Flowers) Pitch 148 Hz, PA 9.3, Ambient (Cirrus) Pitch 577 Hz, PA 10.9, Jazz (Creole Jazz) Pitch 736 Hz, PA 5.3.

The average background noise level in the observation area was found to be $60.7 \mathrm{~dB}$, so the loudspeakers' gain was set accordingly, with the overall aim to exceed the background noise by at least $10 \mathrm{~dB}$ approximately in the whole observation area, as per the simulation in Section 2.2 , so as to provide a reasonably homogeneous signal-to-noise ratio. Figure 3 shows the spectrum of the background noise level recorded on site, compared to the spectra of the three music excerpts, calibrated as if they had an overall sound pressure level of $75 \mathrm{~dB}$. The spectra suggest that the music should be clearly audible over the background noise.

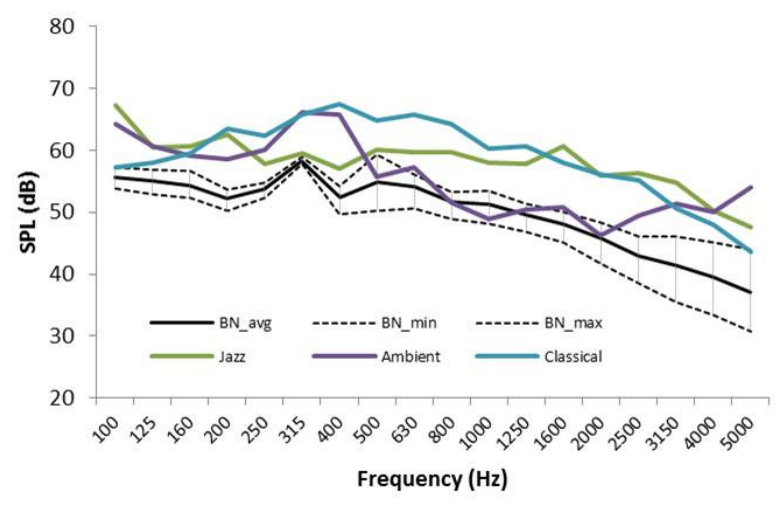

Figure 3. One-third octave band spectrum of the background noise (BN) level (black line) averaged over the ten recording points reported in Figure 2, with minimum and maximum values (dashed lines), compared with the spectra of the three music excerpts (hypothetical broadband sound pressure level of $75 \mathrm{~dB}$ ). 
The music excerpts (reproduced in loop) and the control were cycled repeatedly between 10:00 am and 1:00 pm (or between 11:00 a.m. and 2:00 p.m.) in one-hour slots. The experimental design consisted of $180 \mathrm{~min}$ of exposure for each of the four conditions (i.e., three music excerpts and the control), distributed during the five days of observation. A rotation of the stimuli was set across the week and the daily time slots, in order to obtain the widest range of observations for the same music excerpts in different days and times.

The video recordings were used to investigate peoples' behaviour in response to sound. Direct observational assessment of people behaviours was performed by means of the Behavioural Observation Research Interactive Software (BORIS, v. 2.95, University of Torino, Torino, Italy) [30]. In each BORIS project, information related to a set of observations (such as Behaviours and Participants) are manually annotated through computer vision and respectively inserted in the Ethogram and Subjects table. Ethograms consist of a list of behaviours exhibited by an individual or a group, with corresponding definitions of each using descriptive terms and phrases [31,32]. The ethogram reported in Figure 4 shows the duration of stay (in seconds) of all stopping subjects for a corresponding observation period. For each stopping subject, three more attributes were annotated, namely: (1) Activity (Chatting/Eating-Drinking/Loitering/Smoking/Talking on the phone), related to the main activity carried out by the subjects during their stay; (2) Group (alone/group) related to whether the subjects were staying alone or in group; and (3) Posture (sitting/standing), related to whether the subjects were sitting or standing. Furthermore, the number of passers-by under the bridge was registered in terms of point behaviours (no duration) to examine the total number of subjects passed during the corresponding stimulus condition.

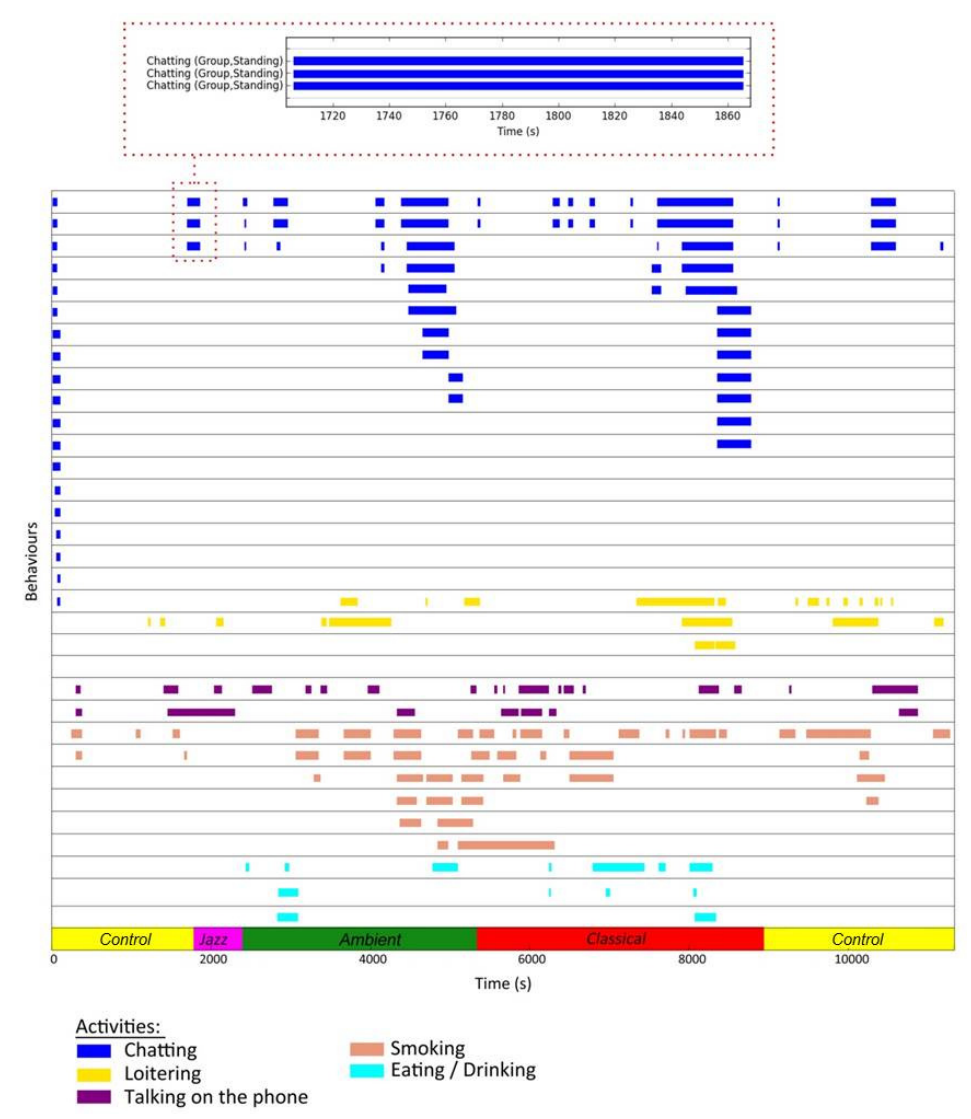

Figure 4. Example of the ethogram obtained from the Behavioural Observation Research Interactive Software (BORIS) exportation. The graph shows the results of a typical daily observation, recording the duration of stay of the subjects during the different excerpts' exposure time and the additional information annotated for each event. 


\section{Results}

\subsection{Effect of Music on Ratio of People Stopping By}

One of the main aims of this research was to investigate whether there is an effect of added music sounds on the number of people stopping by the investigated public area. To address this purpose, two different variables were defined. The fist variable was the Stimulus, consisting of four levels, Classical, Ambient, Jazz music, and Control (No music) condition; while the second variable was defined as the ratio between the number of stoppers by the area and the total number of passers in a given reference time slot; this was defined as a numerical variable, namely the Ratio Stoppers-Passers (RSP). During the experiment, the total exposure time for each Stimulus condition was $180 \mathrm{~min}$. Each of the four overall periods was divided in 18 slots, lasting approximately $10 \mathrm{~min}(180 \mathrm{~min} / 18=10 \mathrm{~min})$, finally obtaining 72 video excerpts $(18 \times 4$ stimuli, including the control $)$. Afterwards, the RSP was calculated and the Stimulus variable was defined for each of the 72 recording slots. Table 1 reports the total number of observations of passers-by and stoppers-by for each stimulus condition.

Table 1. Number of observations according to the different stimuli conditions averaged over the 72 video excerpts. RSP: Ratio of Stoppers-Passers.

\begin{tabular}{ccccc}
\hline Stimulus & Number of Passers-by & Number of Stoppers-by & Mean RSP & Std. Dev. RSP \\
\hline Classical & 4107 & 181 & $4.4 \%$ & $2.5 \%$ \\
Ambient & 2921 & 161 & $5.5 \%$ & $3.8 \%$ \\
Jazz & 2989 & 135 & $4.5 \%$ & $2.9 \%$ \\
Control (No Music) & 2765 & 119 & $4.3 \%$ & $3.5 \%$ \\
\hline
\end{tabular}

The 72 RSP values corresponding to the 72 video excerpts were used for the statistical analysis. Since the distributions of the RSP values across the four stimuli were non-normal, a nonparametric test was used. A Kruskal-Wallis H test was run to determine if there were differences in RSP score between the four conditions. Distributions of RSP scores were not similar for all groups, as assessed by visual inspection of a boxplot. The mean rank of RSP scores was not statistically significantly different between groups, $\chi^{2}(3)=0.24, p=0.971$. Thus, there was no detected effect of the different stimuli on the number of subjects who stopped by the area, nor a significant difference between the RSP values recorded during the music excerpts exposure with respect to the control condition.

\subsection{Effect of Music on Duration of Stay}

Although no significant effect of the added music on the number of people stopping was found, its influence on people's duration of stay was further investigated, and a continuous variable was defined accordingly. Preliminary results reported in Table 2 showed that during the reproduction of the music stimuli, people's duration of stay was longer than in the absence of music. Starting from this point, the influence of three other potential co-variants was explored in order to examine whether people's mean duration of stay was affected by the stimulus variable or other effects.

Table 2. Descriptive statistics of the duration of stay for each stimulus condition.

\begin{tabular}{|c|c|c|c|c|c|}
\hline \multirow{3}{*}{ Stimulus } & \multirow{3}{*}{ Mean Duration of Stay (s) } & \multicolumn{4}{|c|}{ Bootstrap (10,000 Bootstrap Samples) } \\
\hline & & \multirow{2}{*}{ Bias } & \multirow{2}{*}{ Std. Error } & \multicolumn{2}{|c|}{ 95\% Confidence Interval } \\
\hline & & & & Lower & Upper \\
\hline Classical & 320.57 & 0.30 & 24.01 & 274.94 & 368.74 \\
\hline Ambient & 274.69 & -0.18 & 21.74 & 233.42 & 318.60 \\
\hline Jazz & 257.56 & -0.02 & 21.27 & 217.20 & 299.75 \\
\hline Control (No Music) & 132.18 & 0.09 & 11.93 & 109.89 & 156.58 \\
\hline
\end{tabular}




\subsubsection{Analysis of Covariant Attributes on Duration of Stay}

The three attributes described in Section 2.3 (i.e., Activity, Group, and Posture) were considered to be potential covariant factors of influence on the duration of stay. Thus, three corresponding variables were defined. Activity was defined as a categorical variable with five levels: Chatting, Eating-Drinking, Loitering, Smoking, and Talking on the phone. Group was defined as a dichotomous variable: No (Alone) or Yes (Two or more people). Posture was defined as a dichotomous variable: Standing or Sitting.

Since the distribution of duration of stay scores was positively skewed, a log transform (natural logarithm) was performed to normalise the data, and this resulted in an approximately normal distribution of scores. Thus, from now on, the Duration of stay variable will refer to the log-transformed dataset.

A one-way Welch ANOVA was carried out to investigate whether the different activities carried out affected people's mean duration of stay. Homogeneity of variances was violated, as assessed by Levene's Test of Homogeneity of Variance $(p=0.018)$. Duration of stay score was statistically significantly different between different activities groups, Welch's $F(4,143.522)=14.821, p=0.001$, partial $\eta^{2}=0.030$. However, Games-Howell post hoc analysis revealed that only the duration of Talking on the phone events $(\mathrm{M}=4.66, \mathrm{SD}=1.06)$ had a statistically significant difference with respect to Smoking events $(M=5.26, S D=0.96), p=0.04$, and Eating/Drinking events $(M=5.38, S D=1.14)$, $p=0.04$. It seems likely to assert that the occurrence of Talking on the phone events implies a shorter mean duration of stay than the one detectable during the other four activities. A second critical inspection of the recordings revealed that in most cases, the duration of the Talking on the phones event was mainly related to the actual duration of the call (i.e., people would stay in the observation area only for the time necessary for the call, being uninterested in the music). No significant difference was observed between the duration of stay recorded during Chatting, Eating/Drinking, Loitering, and Smoking events, so no other relative effect could be stated according to the Activities variable.

Furthermore, a one-way Welch ANOVA was carried out to investigate whether the being alone or in group could affect people's mean duration of stay. Homogeneity of variances was not violated in this case, as assessed by Levene's Test of Homogeneity of Variance $(p=0.050)$, but the difference between the two levels of the Group variable was not statistically significant, Welch's $F(1,577.373)=0.070$, $p=0.791$. Eventually, a one-way Welch ANOVA was carried out for the Posture variable, to investigate whether standing or sitting could affect people's mean duration of stay. Homogeneity of variances was violated, as assessed by Levene's Test of Homogeneity of Variance $(p=0.004)$. The difference between the two levels of the Posture variable was statistically significant, Welch's $F(1,469.457)=136.946$, $p<0.001$, partial $\eta^{2}=0.183$.

Thus, the Activity and Group variables were excluded from further analysis, as their influence on the duration of stay values was not significant. Only the effects of Posture and Stimulus on the Duration of stay variable will be explored in the following section.

\subsubsection{Combined Effect of Posture and Music on Duration of Stay}

A two-way ANOVA was conducted to examine the effects of Posture and Stimulus on Duration of stay. Homogeneity of variances was assessed by Levene's test, and it was violated $(p<0.001)$; however, there is an overall consensus in the literature to carry on regardless when the ratio of the largest to the smallest group variance is less than three (a condition that is met in this study), since the test is reasonably robust to heterogeneity of variance in these cases [33].

The interaction effect between Posture and Stimulus was not statistically significant for Duration of stay scores, $F(3,574)=1.197, p=0.310$, partial $\eta^{2}=0.006$. Therefore, an analysis of the main effect for Stimulus was performed, which indicated that the main effect was statistically significant, $F(3,574)=3.781, p=0.010$, partial $\eta^{2}=0.019$. All pairwise comparisons were run, where reported $95 \%$ confidence intervals and $p$-values were Bonferroni-adjusted. The unweighted marginal means of Duration of stay scores for Classical, Ambient, Jazz, and Control (No music) were $5.29 \pm 0.072$, 
$5.25 \pm 0.079,5.17 \pm 0.087$, and $4.83 \pm 0.120$, respectively. These point scores of the log-transformed duration variable correspond to mean Duration of Stay of 198 seconds (Classical), $190 \mathrm{~s}$ (Ambient), $176 \mathrm{~s}$ (Jazz), and $125 \mathrm{~s}$ (Control). Figure 5 shows the estimated marginal means of the ANOVA model for the log-transformed version of Duration of stay. The Control condition was associated with a mean Duration of stay score 0.45 points lower than Classical $(p=0.007), 0.42$ points lower than Ambient $(p=0.021)$, and 0.34 points lower than Jazz, but the latter difference was not statistically significant $(p=0.124)$. For the sake of clarity, Figure 6 reports the mean Duration of stay scores (as observed during the on-site campaign).

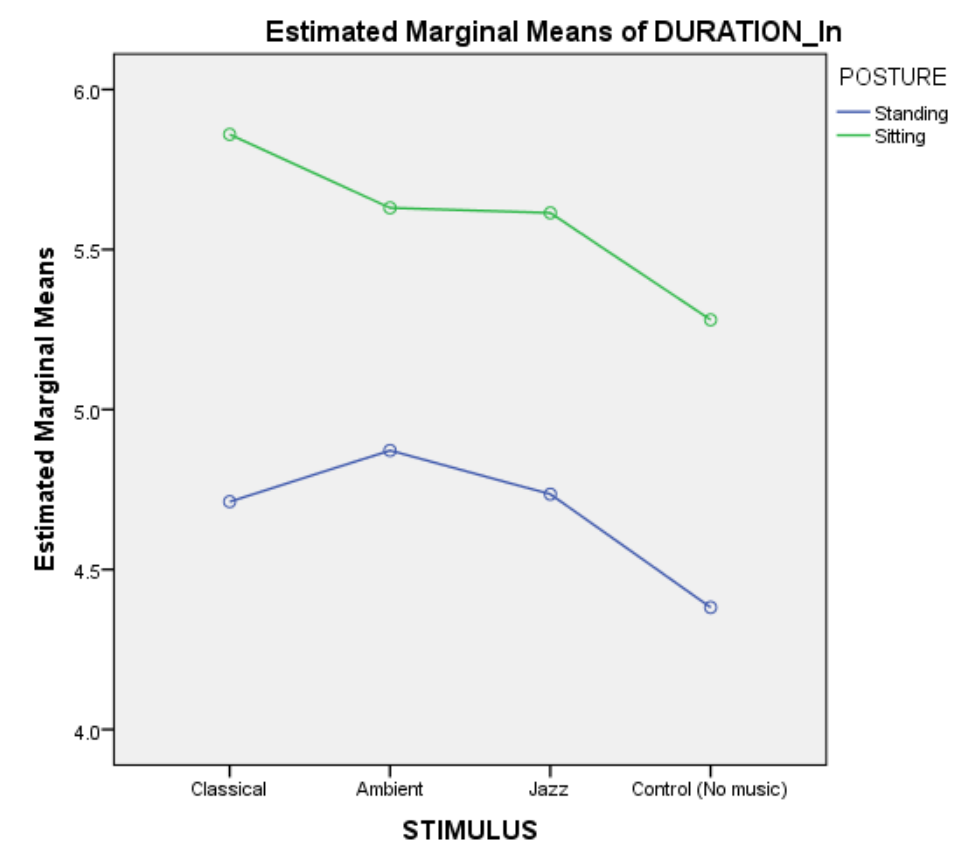

Figure 5. Mean Duration of stay (log-transformed) as a function of the Stimulus variable, when controlling for the Posture variable.

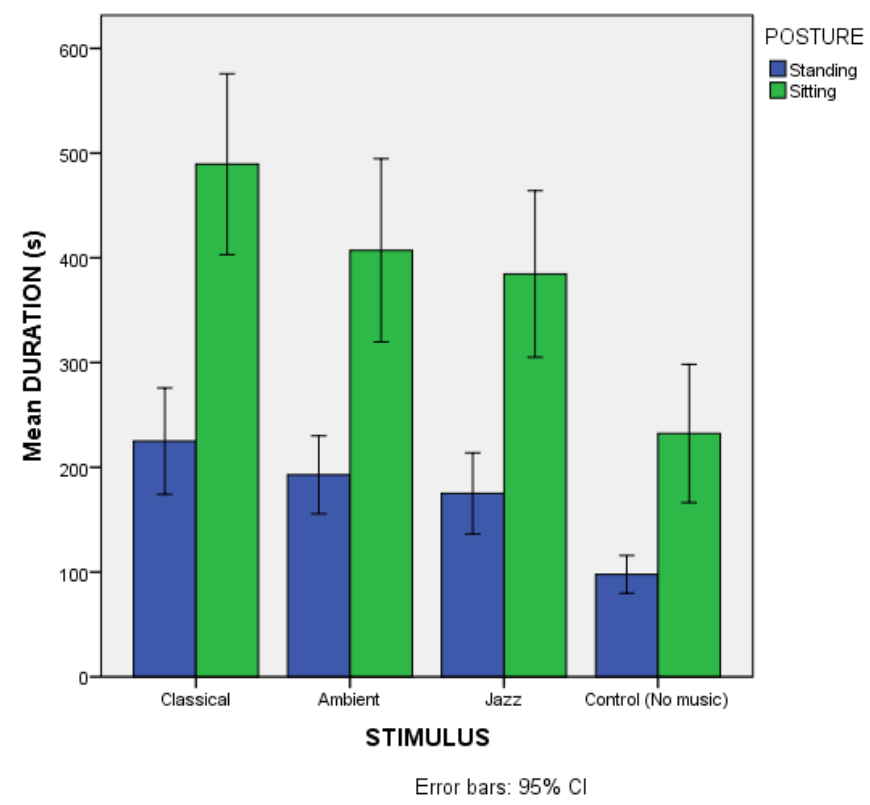

Figure 6. Mean Duration of stay (as observed) as a function of the Stimulus variable, for both the Standing and Sitting conditions. 
Thus, for the investigated case, the above findings seem to suggest that music-without any kind of interaction with other additional qualitative variables-had the capacity to positively affect people's duration of stay in the observation area.

\section{Discussion and Conclusions}

An experiment based on covert behavioural observations was performed in a typical open public space in Sheffield, mainly used as a connection between different University buildings. Three musical excerpts (and a control condition with no music) were reproduced cyclically to test whether there is an effect of background music on the number of persons stopping in the urban space and if added sounds could affect people's duration of stay in the investigated area. Overall, the experiment reported in this paper led to two main conclusions:

- the presence of music did not affect the number of subjects stopping by the observed area; thus, the manipulation of the sound environment had no power to influence people's choice about stopping or not in the space;

- the presence of background music influenced people's mean duration of stay in the public space, with added sound (Classical and Ambient music in the investigated case) implying a longer duration of stay than the one recorded in the control (no music) condition.

The observation site considered in this research is a space with apparently no specific function other than transit; i.e., people do not "go there" as a destination. It can be considered as a place where people "stop for a moment" on their route from one point to another, which would justify the lack of an overall effect of music on people's decision to stop or go. Within the framework of this study, it was assumed that the Duration of stay variable could be used as a proxy for a measure of perceived safety and relaxation. That is, if people decide to stay longer in an apparently unattractive place, to some extent, it can be implied that they feel safe and relaxed there [6]. This is connected to the ongoing debate about outcomes and preference in soundscape studies. Brown et al. [12] suggested that people's preferred outcome with respect to the acoustic environment is context-dependent. They reported a broad spectrum of soundscape outcomes, which are likely to be related to different soundscape appreciation and preference in different contexts. For example, the desired soundscape of urban parks might be assessed against calmness or restorativeness criteria (e.g., [34]), while for downtown or commercial environments, liveliness and excitement dimensions might provide more useful insights into the soundscape assessment of such places [16]. Thus, it was considered that perceptual dimensions related to safety, sense of control, or relaxation might be more relevant for the investigated context. The outcomes of this research could be a potential complement to the existing Masterplan proposal based on traditional urban renewal practices [15]; sound as well as light or green features should be an actual tool within the broader framework of urban planning and design policies.

There are of course some limitations, which could not be controlled for. For instance, during the experimental sessions, it was not possible to precisely monitor the signal-to-noise ratio (SNR) in the observation area. Thus, in spite of the efforts to achieve a homogenous sound field during the reproduction of the stimuli, it is likely that people were exposed to slightly different sound levels, depending on where they were stopping. However, strictly controlled SNR conditions might only be achieved in laboratory experiments, the ecological validity of which has been a topic long debated in soundscape research with no clear consensus so far (e.g., [35]). Furthermore, there might have been other confounding factors affecting the duration of stay in the observation area (e.g., personal attitudes, music preference, etc.). However, given the nature of this study (i.e., covert observational research), such information was not to be sought from observed participants (e.g., through questionnaires) in order to not bias the main investigated variable.

Overall, these limitations are the price this study pays when trying to overcome a significant methodological issue for soundscape assessment in public outdoor contexts that Brown et al. [12] for soundscape research defined as the "experimenter effect" where "... measurement of people's preference 
in these situations using questionnaire methods requires first drawing their attention to something upon which they may never have consciously reflected."

Taken together, the results of this study suggest that added music in public spaces might have the capability to increase the liveability and the pleasantness of places that only have practical functions (e.g., transit, waiting). Soundscape development and design can promote the attractiveness of a public space through its ability to increase the perceived quality and positively impact the psychological and physiological wellbeing of citizens, and it can help to build social cohesion amongst users and define the social use values of city spaces.

Acknowledgments: This research received funding through the People Programme (Marie Curie Actions) of the European Union's 7th Framework Programme FP7/2007-2013 under REA grant agreement n² 290110, SONORUS "Urban Sound Planner". The authors are grateful to Pierluigi Ciullo for his support in data collection, to James Uttley for his comments on the preliminary version of the manuscript and to the anonymous reviewers for their useful and constructive insights.

Author Contributions: F.A., F.L., E.K.-K., J.K. and A.A. conceived and designed the experiments; F.A., F.L. and E.K.-K. analyzed the data; F.A., F.L., E.K.-K., J.K. and A.A. wrote the paper.

Conflicts of Interest: The authors declare no conflict of interest.

\section{References}

1. Schafer, R.M. The Tuning of the World; Knopf: New York, NY, USA, 1977.

2. International Organization for Standardization. ISO 12913-1:2014 Acoustics_-Soundscape_Part 1: Definition and Conceptual Framework; ISO: Geneva, Switzerland, 2014.

3. Areni, C.S.; Kim, D. The influence of background music on shopping behavior: Classical versus top-forty music in a wine store. Adv. Consum. Res. 1993, 20, 336-340.

4. Yalch, R.F.; Spangenberg, E.R. The effects of music in a retail setting on real and perceived shopping times. J. Bus. Res. 2000, 49, 139-147. [CrossRef]

5. Milliman, R.E. Using background music to affect the behavior of supermarket shoppers. J. Mark. 1982, 46, 86-91. [CrossRef]

6. Sayin, E.; Krishna, A.; Ardelet, C.; Decré, G.B.; Goudey, A. "Sound and safe": The effect of ambient sound on the perceived safety of public spaces. Int. J. Res. Mark. 2015, 32, 343-353. [CrossRef]

7. Kampfe, J.; Sedlmeier, P.; Renkewitz, F. The impact of background music on adult listeners: A meta-analysis. Psychol. Music 2011, 39, 424-448. [CrossRef]

8. Ryuma Kuribayashi, R.; Nittono, H. Speeding up the tempo of background sounds accelerates the pace of behavior. Psychol. Music 2015, 43, 808-817. [CrossRef]

9. Easteal, M.; Bannister, S.; Kang, J.; Aletta, F.; Lavia, L.; Witchel, H.J. Urban sound planning in Brighton and Hove. In Proceedings of the Forum Acusticum, Krakow, Poland, 7-12 September 2014.

10. Witchel, H.J.; Lavia, L.; Westling, C.E.I.; Healy, A.; Needham, R.; Chockalingam, N. Using body language indicators for assessing the effects of soundscape quality on Individuals. In Proceedings of the AIA-DAGA Conference on Acoustics, Merano, Italy, 18-21 March 2013.

11. Schafer, T.; Huron, D.; Shanahan, D.; Sedlmeier, P. The sounds of safety: Stress and danger in music perception. Front. Psychol. 2015, 6, 1-12. [CrossRef] [PubMed]

12. Brown, A.L.; Kang, J.; Gjestland, T. Towards standardization in soundscape preference assessment. Appl. Acoust. 2011, 72, 387-392. [CrossRef]

13. Department for Communities and Local Government. Planning Policy Statement 3 (PPS3): Housing; Department for Communities and Local Government: London, UK, 2006.

14. Dempsey, N. Are good-quality environments socially cohesive? Measuring quality and cohesion in urban neighbourhoods. Town Plan. Rev. 2009, 80, 315-345. [CrossRef]

15. University of Sheffield. Available online: https://www.sheffield.ac.uk/campusmasterplan (accessed on 24 July 2016).

16. Aletta, F.; Kang, J.; Axelsson, O. Soundscape descriptors and a conceptual framework for developing predictive soundscape models. Landsc. Urban Plan. 2016, 149, 65-74. [CrossRef] 
17. Lòpez-Méndez, A.; Westling, C.E.I.; Emonet, R.; Eastel, M.; Lavia, L.; Witchel, H.J.; Odobez, J.-M. Automated bobbing and phase analysis to measure walking entrainment to music. In Proceedings of the International Conference on Image Processing, Paris, France, 23-30 October 2014.

18. Yang, W.; Kang, J. Acoustic comfort evaluation in urban open public spaces. Appl. Acoust. 2005, 66, 211-229. [CrossRef]

19. Stockfelt, T. Sound as an existential necessity. J. Sound Vib. 1991, 151, 367-370. [CrossRef]

20. Davies, W.J.; Adams, M.D.; Bruce, N.S.; Cain, R.; Carlyle, A.; Cusack, P.; Hall, D.A.; Hume, K.I.; Irwin, A.; Jennings, P.; et al. Perception of soundscapes: An interdisciplinary approach. Appl. Acoust. 2013, 74, $224-231$. [CrossRef]

21. Yang, M.; Kang, J. Pitch features of environmental sounds. J. Sound Vib. 2016, 374, 312-328. [CrossRef]

22. Raimbault, M.; Lavandier, C.; Ėrengier, M.B. Ambient sound assessment of urban environments: Field studies in two French cities. Appl. Acoust. 2003, 64, 1241-1256. [CrossRef]

23. Axelsson, Ö.; Nilsson, M.E.; Berglund, B. A principal components model of soundscape perception. J. Acoust. Soc. Am. 2010, 128, 2836-2846. [CrossRef] [PubMed]

24. Moore, B.C.J. An Introduction to the Psychology of Hearing; Academic Press: London, UK, 1997.

25. American Standards Association. American Standard Acoustical Terminology (Including Mechanical Shock and Vibration; Acoustical Society of America: New York, NY, USA, 1960.

26. Hevner, K. The affective value of pitch and tempo in music. Am. J. Psychol. 1937, 49, 621-630. [CrossRef]

27. Balkwill, L.L.; Thompson, W.F. Across-cultural investigation of the perception of emotion in music: Psychophysical and cultural cues. Music Percept. 1999, 17, 43-64. [CrossRef]

28. Krumhansl, C.L. An exploratory study of musical emotions and psychophysiology. Can. J. Exp. Psychol. 1997, 51, 336-352. [CrossRef] [PubMed]

29. Ellis, A.J.; Mendel, A. Studies in the History of Music Pitch; Frits Knuf: New York, NY, USA, 1968.

30. Behavioral Observation Research Interactive Software (BORIS) version 2.95 User Guide. Available online: http:/ / www.boris.unito.it (accessed on 24 July 2016).

31. Martin, P.; Bateson, P. Measuring Behaviour: An Introductory Guide; Cambridge University Press: New York, NY, USA, 2007.

32. Stanton, L.A.; Sullivan, M.S.; Fazio, J.M. A standardized ethogram for the felidae: A tool for behavioural researchers. Appl. Anim. Behav. Sci. 2015, 173, 3-16. [CrossRef]

33. Jaccard, J. Interaction Effects in Factorial Analysis of Variance; Sage Publications: Thousand Oaks, CA, USA, 1998.

34. Payne, S.R. The production of a perceived restorativeness soundscape scale. Appl. Acoust. 2013, 74, $255-263$. [CrossRef]

35. Guastavino, C.; Katz, B.F.; Polack, J.; Levitin, D.J.; Dubois, D. Ecological validity of soundscape reproduction. Acta Acust. United Acust. 2005, 91, 333-341.

(C) 2016 by the authors; licensee MDPI, Basel, Switzerland. This article is an open access article distributed under the terms and conditions of the Creative Commons Attribution (CC-BY) license (http://creativecommons.org/licenses/by/4.0/). 